E X P E R I E N C I A S

\title{
Una experiencia para atender a la diversidad en la universidad
}

\section{An experience of atenttion to diversity at university}

Remedios Benítez Gavira ${ }^{1}$, Sonia Aguilar Gavira², Laura de las Flores Sánchez Calleja ${ }^{3}$

Recibido: 20 de abril de 2019 Aceptado: 13 de junio de 2019 Publicado: 31 de julio de 2019

To cite this article: Benítez, R., Aguilar, S. y Sánchez, L. (2019). Una experiencia para atender a la diversidad en la universidad. Márgenes, Revista de Educación de la Universidad de Málaga, o (o), 76-96

DOI: http://dx.doi.org/10.24310/mgnmar.voio.6230

\author{
${ }^{1}$ Remedios Benítez Gavira 0000-0001-6937-9221 \\ Departamento de Didáctica, Universidad de Cádiz \\ r.benitez@uca.es
}
${ }^{2}$ Sonia Aguilar Gavira 0000-0002-4168-271X
Departamento de Didáctica, Universidad de Cádiz
sonia.aguilar@uca.es

\author{
${ }^{3}$ Laura de las Flores Sánchez Calleja 0000-0003-2174-0369 \\ Departamento de Didáctica, Universidad de Cádiz \\ lauradelasflores.sanchez@uca.es
}

\section{RESUMEN}

Este trabajo muestra una experiencia llevada a cabo en la asignatura de cuarto curso del grado de Educación Infantil denominada "Fundamentos Pedagógicos de las Necesidades Educativas en la Infancia". Nuestra principal intención es atender de la forma más adecuada al alumnado universitario ofreciéndole a su vez estrategias inclusivas de forma vivenciada que puedan transferir con los ajustes necesarios a sus aulas en el futuro. Para ello, estudiamos distintos modelos metodológicos con los siguientes criterios: que se adecuaran a nuestro contexto, que respetaran los distintos ritmos de aprendizaje, que ayudaran en la gestión de las grandes ratios, que posibilitaran responder a los intereses del alumnado y que a su vez potenciaran el conocimiento interpersonal, pues una de las necesidades que manifiesta el alumnado es que después de cuatro años conviviendo en un aula, no se conocen. Así, realizamos una revisión bibliográfica en relación a las agrupaciones y metodologías favorecedoras de la atención a la diversidad marcándonos como objetivos: atender a las necesidades e intereses del alumnado, promover el encuentro y vivenciar estrategias inclusivas.

Palabras clave: Diseño Universal de Aprendizaje; formación inicial del profesorado; Educación Infantil; atención a la diversidad

\section{ABSTRACT}

This work shows an experience carried out in the subject of the fourth year of the Early Childhood Education degree called "Pedagogical Fundamentals of Educational Needs in Childhood". Our main intention is to attend in the most adequate way to the university students offering at the same time inclusive strategies in an experienced way that they can transfer with the necessary adjustments to their classrooms in the future. To do this, we studied different methodological models with the following criteria: that they adapt to our context, that they respect the different learning rhythms, 


\section{$\begin{array}{lllllllllllll} & \text { E } & \mathbf{X} & \mathbf{P} & \mathbf{E} & \mathbf{R} & \mathrm{I} & \mathrm{E} & \mathbf{N} & \mathrm{C} & \mathrm{I} & \mathrm{A} & \mathrm{S}\end{array}$}

that will help in the management of the great ratios, that will make it possible to respond to the interests of the students and that in turn enhance interpersonal knowledge, because one of the needs expressed by students is that after four years living in a classroom they do not know each other. Thus, we conducted a literature review in relation to groups and methodologies favoring attention to diversity, marking us as objectives: to meet the needs and interests of students, promote the meeting and experience inclusive strategies.

Keywords: Universal Design of Learning; initial teacher education; child education; attention to diversity

\section{INTRODUCCIÓN}

La diversidad es una fuente de enriquecimiento que en muchas ocasiones no se potencia y a veces, no se atiende desde la universidad. Los grupos-clase son divididos en subgrupos para reducir las ratios y esta realidad está provocando que muchos de nuestros estudiantes terminen el Grado de Educación Infantil sin conocerse. Sin embargo, son diversos los trabajos que nos muestran que es muy importante conocernos para aprender en un buen clima de respeto (Bravo y Herrera, 2011; González, Díez, López y Román, 2010). Desde aquí y con las circunstancias concretas encontradas en nuestro contexto es importante realizar un diseño didáctico inclusivo. Y entonces, aparece el siguiente interrogante, ¿cómo hacerlo?

Partiendo de la afirmación de Tomlinson (2005, p. 27) "una "buena" educación [...] ayuda a los alumnos a maximizar su capacidad de aprendizaje" y entendiendo que "cada persona es única e irrepetible, diferente de las demás [...] ello es lo que hace que cualquier grupo de personas, por muy pequeño que sea, sea heterogéneo" (Benítez y Aguilar, 2015, p. 98).

Según Tomlinson (2013) en las aulas diversificadas el profesorado parte del momento en el que se encuentra su alumnado, apostando por la diversidad existente en la misma, proporcionando a cada individuo lo que necesita, desde la creencia de construir su máximo aprendizaje, pidiéndole un trabajo duro, para alcanzar metas por encima de sus propias expectativas y para lo que es necesario esfuerzo, riesgo y éxito personal. En ocasiones, el alumnado no confía en sus posibilidades y no piensa conseguir metas a priori que finalmente pueden alcanzar. Para ello, el profesorado observa activamente a sus estudiantes y pone a su servicio los recursos para ajustar las clases a cada individuo, dado que el profesorado cree en las posibilidades del alumnado y sienten que son capaces, es decir, tiene confianza en su desarrollo $\mathrm{y}$ busca sus potencialidades para ir desde lo que funciona bien a lo que no funciona tan bien (Tomlinson, 2005). Algo muy diferente a lo que se realiza desde un modelo integrador, centrado en las limitaciones y déficits.

Desde esta premisa y como responsables de la formación inicial de los futuros y futuras docentes de Educación Infantil, consideramos de suma transcendencia andamiar de forma práctica y experiencial la construcción de una mirada más humana y respetuosa hacia la diversidad, no como algo extraordinario y especial, sino como algo natural que nos ofrece grandes oportunidades de crecimiento y desarrollo desde una perspectiva más amplia y enriquecedora. Aunque el eje central de este 


\section{$\begin{array}{lllllllllllll}\text { E } & \mathbf{X} & \mathbf{P} & \mathbf{E} & \mathbf{R} & \mathrm{I} & \mathrm{E} & \mathbf{N} & \mathrm{C} & \mathrm{I} & \mathrm{A} & \mathrm{S}\end{array}$}

trabajo sea una experiencia, ésta no podría ser desarrollada sin unos fundamentos teóricos que constituyen los cimientos de toda acción educativa con sentido y a su vez permiten la construcción de infinidad de posibilidades para ofrecer una respuesta ajustada, a la realidad concreta de las personas que componen el grupo-clase en el que nos encontremos.

\section{2. ¿QUÉ FUNDAMENTOS TEÓRICOS SUSTENTAN LA EXPERIENCIA DESARROLLADA?}

Son tres los fundamentos que sirven de sostén teórico a la experiencia desarrollada, y que serán expuestos a lo largo de este apartado. Concretamente, se trata de: las aulas diversificadas, el Diseño Universal para el Aprendizaje y la Neurodidáctica. Entre estos fundamentos se establecen conexiones desde los planteamientos y principios pedagógicos que se desprenden de sus postulados como se mostrará a continuación en la exposición de los mismos.

\subsection{Aulas diversificadas}

Las aulas diversificadas nos van a permitir realmente atender a la diversidad del grupo desde la individualidad de cada persona que lo compone, en pos de construir una intervención educativa de corte inclusivo, desde el hecho de establecer unas metas alcanzables para ir elevando los objetivos, testando los límites de cada sujeto, proporcionando para ello la figura docente un clima de seguridad y confianza, guiándoles hacia un desarrollo pleno de sus potencialidades, así como alabar y reconocer los intentos y logros conseguidos (Tomlinson, 2005). Y es que como afirma Carpena (2015) "se establecen conexiones neuronales importante que contribuirán al desarrollo cerebral, cuando los retos son dirigidos a objetivos claros y alcanzables según las características personales" (p. 140). Estas aulas, se basan en una enseñanza que, según Tomlinson (2005) ha de ser:

- Proactiva y no reactiva. Huye de un enfoque único para todo el grupo-clase y cuando no funciona busca una solución. Se anticipa a ello, ofreciendo diversidad de fuentes y un enfoque múltiple para la construcción y expresión del propio aprendizaje, así como, diversos agrupamientos: gran grupo, pequeños grupos, o de forma individual y pluralidad de tareas. No es necesario hacer lo mismo al mismo tiempo, es enriquecedor hacer cosas distintas con diferentes canales o vías de aprendizaje que ayuden a conectar con los estudiantes (CAST, 2011).

- Cualitativa y no cuantitativa. No consiste en ofrecer más tareas sino en adecuar las tareas a la propia realidad a la que van dirigida, teniendo en cuenta su propia idiosincrasia ajustándola a las necesidades del aula.

- Centrada en el alumnado y no en el profesorado, buscando dar respuestas a los intereses y necesidades de cada una de las personas que componen el grupoclase realizando para ello planteamientos estimulantes y atractivos.

- Dinámica y no estática, partiendo de la idea de que el conocimiento está en construcción, vivo, que fluye y evoluciona (Pérez, 2014). Además, los agentes impli- 
cados en el proceso de enseñanza-aprendizaje están activos en el sentido de que aprenden de forma conjunta. Concretamente el alumnado desde la construcción de un aprendizaje significativo y relevante, y el profesado desde la observación, supervisión y realización de los ajustes necesarios a lo largo del todo el proceso de aprendizaje para que cada alumno y alumna saque lo mejor de sí. Aunque no existe una fórmula mágica para crear un aula diversificada, Tomlinson (2001) nos muestra una serie de principios, ideas o pasos que guían dicha diversificación:

- El profesorado se centra en lo esencial, en lo que considera relevante para el aprendizaje del alumnado.

- Apreciar las diferencias entre el alumnado tomándose como valor para el proceso de enseñanza-aprendizaje.

- Durante todo el proceso de enseñanza se lleva a cabo una evaluación continua, permitiéndonos a la figura docente reflexionar y realizar las modificaciones oportunas con el objetivo de mejorar el aprendizaje del alumnado y nuestra propia práctica.

- Ajustar en función de las vías de aprendizaje del alumnado, sus ritmos e intereses tanto los contenidos como los procesos y los productos.

- Todos los estudiantes participan en tareas adecuadas para ellos y ellas.

- El profesorado y el estudiante colaboran en el aprendizaje y son partícipes activos del mismo.

- El método de trabajo debe ser en todo momento flexible. Las aulas diversificadas difieren bastante con la enseñanza tradicional dado que su intención máxima es ofrecer como hemos dicho anteriormente la atención necesaria al alumnado. Para ello, Parafraseando a Tomlinson (2001) podemos ver algunos aspectos que la diferencia de dicha enseñanza tradicional:

- Mientras que en una enseñanza tradicional las diferencias del alumnado se plantean o se entienden como problema, en las aulas diversificadas se estudian y analizan como base para la planificación.

- La enseñanza tradicional parte de la evaluación final del proceso para conocer quien ha aprendido utilizando solo una única forma para todos y todas, en lugar de lo que ocurre en las aulas diversificadas que entienden la evaluación continua como una herramienta para comprender cómo las instituciones pueden mejorar en la atención de las necesidades del alumnado.

- La enseñanza tradicional solo conoce una única inteligencia mientras que las aulas diversificadas parten del concepto de que existen múltiples inteligencias.

- La excelencia es entendida en sentido único por la enseñanza tradicional mientras que para las aulas diversificadas comienza en un punto de partida y desde ahí se marca.

- La primera no tiene en cuenta los intereses del alumnado mientras que para la segunda habitualmente es la base para crear la planificación de los futuros aprendizajes y los perfiles de aprendizaje. En palabras de Díez y Huete (1997): 
No significa adoptar medidas especiales para el alumnado problemático, sino adoptar un modelo de desarrollo del currículo que facilite el aprendizaje del alumnado en su diversidad. Tampoco es una cuestión de cantidad sino de calidad; una actitud y una convicción cultural antes que un recurso técnico, que requiere enseñar a cada uno de forma diferenciada (pp.15-17).

- La enseñanza tradicional se basa en la instrucción para todo el grupo clase, en lugar de ofrecer diferentes alternativas o disposiciones distintas para la misma. Además, dicha instrucción está basada o dirigida por el profesorado, mediante libros de texto y con un único libro. En el caso de las aulas diversificadas se basa en los intereses, ritmos y perfiles del alumnado utilizando diversidad de materiales para responder al grupo-clase. Mientras que en la enseñanza tradicional se plantea una única tarea para todos y todas, la enseñanza diversificada plantea múltiples opciones.

- La base de la enseñanza, en la escuela tradicional, son hechos y destrezas descontextualizadas, no utiliza destrezas para promover la comprensión y dar sentido a los conceptos clave "aulas diversificadas".

- Los sucesos en la enseñanza tradicional solo tienen una interpretación, mientras que a través de las aulas diversificadas se buscan diferentes perspectivas de las ideas, de los sucesos y hechos.

- El tiempo se flexibiliza en relación a las necesidades del alumnado en las aulas diversificadas, algo que no es así en las aulas tradicionales.

- El rol del profesorado también cambia en relación a las dos enseñanzas. En la escuela tradicional el profesorado dirige al alumnado, resuelve los problemas y especifica los estándares de evaluación de toda la clase. Por el contrario, en las aulas diversificadas, el profesorado actúa como guía o facilitador de las destrezas para aumentar su autoconfianza en la construcción de su aprendizaje, el profesorado ayuda a la creación de redes de trabajo entre iguales para la resolución de problemas y el alumnado trabaja con el profesorado para crear sus propios estándares de evaluación además de los de la clase.

En síntesis, toda institución educativa "debe procurar, [...] el desarrollo singular, en cada individuo, de estas cualidades, capacidades o competencias, como sistemas complejos de comprensión y actuación, que incluyen al mismo nivel y con la misma relevancia: conocimientos, habilidades, emociones, actitudes y valores, conscientes e inconscientes" (Pérez, 2014, p. 39). Y a la vez fomentar el trabajo cooperativo y la ayuda mutua pues, la relación con la otra persona es una fuente de enriquecimiento fundamental. No olvidando que el proceso de enseñanza-aprendizaje es, en esencia, una interacción entre personas donde lo emocional constituye un pilar básico (Bächler y Pozo, 2016). Así se refleja en palabras de Ibarrola (2016) cuando manifiesta que "nuestra capacidad para aprender se encuentra profundamente arraigada en las relaciones. Nuestro rendimiento en el aprendizaje puede verse profundamente afectado por el entorno emocional en el cual se produce ese aprendizaje" (p. 264). 


\subsection{Diseño Universal para el Aprendizaje}

Para alcanzar esa atención a la diversidad a la que nos referimos en el apartado anterior, es primordial, la eliminación de barreras que puedan dificultar al sujeto acceder al aprendizaje y a la participación. Entendiendo que dichas barreras no se encuentran en el alumnado sino en la forma en la que el profesorado enseña y en el contexto donde se desenvuelve desde el momento en que le ofrecemos la bienvenida en el aula. Es por ello, que otro pilar básico es el Diseño Universal de aprendizaje (en adelante DUA), el cual supone "una visión de la intervención educativa para que todo el alumnado tenga cabida en los procesos de enseñanza, en su planificación y en su desarrollo, a través de diseños curriculares flexibles que tengan en cuenta la diversidad" (Alba, 2018, p.21). Es necesaria una transformación dentro de los contextos universitarios, donde la formación inicial del profesorado les permita desarrollar estrategias de intervención, es decir, conocer y vivenciar como futuros profesionales como dar respuesta a la diversidad existente en las aulas y para lo que el conocimiento y capacitación en el uso de herramientas como el DUA, favorecerá que en su futura labor docente facilite la accesibilidad, participación y experiencias de aprendizaje de todo su alumnado. No podemos olvidarnos que el alumnado universitario es el principal agente de cambio de un modelo de enseñanza tradicional persistente en el tiempo y en el que parte del alumnado ha venido siendo excluido por no adaptarse al sistema; aunque para alcanzar dicho cambio es necesario formar a buenos y buenas docentes. De acuerdo con Tonucci (2017):

las leyes no son capaces de modificar las praxis, las leyes no son capaces de modificar la escuela. Una buena ley nunca será capaz de inducir a un mal maestro a hacer una buena escuela, ni nunca una mala ley podrá obligar a un buen maestro a hacerlo mal (p.12).

Desde el inicio, la práctica educativa debe diseñarse pensando en todos y todas, eliminando cualquier barrera que pueda hacer de los entornos o las prácticas situaciones discapacitantes para algunas personas. Si comenzamos nuestra planificación ajustando nuestros materiales, entornos, herramientas y contenidos para que sean accesibles para todas las personas y donde las metodologías, metas y método de evaluación respondan a dicha diversidad, las barreras irán limándose y el acceso, la participación y el aprendizaje cobrarán un sentido mucho más inclusivo en nuestras aulas. Accesibilidad entendida como una condición imprescindible para garantizar la equidad en el aula, favoreciendo la misma, espacios inclusivos (Giné y Font, 2007; CAST, 2011). Como podemos ver el enfoque del DUA responde a los principios de una educación inclusiva, ya que previamente, en el periodo planificador del proceso educativo se tiene en cuenta las diferencias personales, necesidades e intereses de todo el alumnado.

Aclarar que el DUA no significa "reducir, simplificar o "rebajar" los contenidos de aprendizaje para algunos estudiantes o buscar el "mínimo contenido común". Es, en definitiva, hacer el aprendizaje accesible para todos y todas" (Simón et al., 2016, p.12) permitiendo que cada alumno/a elija la opción que mejor favorece su aprendizaje y no solo para aquellas personas que presentan necesidades educati- 
vas especiales o necesidades de apoyo educativo. Si algo caracteriza al DUA es que "rompe la dicotomía entre alumnado con discapacidad y sin discapacidad. La diversidad es un concepto que se aplica a todos los estudiantes, que tienen diferentes capacidades que se desarrollan en mayor o menor grado, [...]" (Alba, Sánchez y Zubillaga, 2014, p.11).

De este modo el DUA se convierte en parte de esa apuesta transformadora por el cambio social hacia una educación más inclusiva en la que se favorece el proceso de un currículo rígido y único a otro flexible e inclusivo (Alba, 2016). En este cambio advierten los y las autoras que el DUA trasciende lenguas, culturas, diferencias y dificultades convirtiéndose en una vía eficaz para eliminar o superar aquellas barreras que encontramos en el aprendizaje. Si la educación es un derecho de todos y todas, el DUA se convierte en una opción imprescindible al servicio de educadores, educadoras y estudiantes.

De acuerdo con Alba (2018) es algo asiduo que en el ámbito educativo se confunda "igualdad de oportunidades con igualdad de situaciones de aprendizaje y se propone una enseñanza basada solo en actividades homogéneas" (p.22) para todo el alumnado. Incorporar en nuestra práctica docente diferentes metodologías, así como el DUA, nos va a permitir atender a las distintas motivaciones e intereses, estilos de aprendizaje o inteligencias del alumnado. Para ello, hay que tener en cuenta los tres principios en los que se sustenta el DUA, como: 1) El profesorado debe aportar múltiples formas de representación de la información y los contenidos, puesto que el alumnado difiere en cuanto a la forma de percibir y comprender la información. Esto favorece que el alumnado logre las competencias necesarias para construir de forma activa su propio conocimiento como expertos de su aprendizaje, ya que le permite disponer y conocer cuáles son los recursos que más se ajustan a sus características personales y "permitiría que todo el alumnado se viera identificado en el proceso de enseñanza” (Aguilar y Benítez, 2017, p. 5); 2) Aportar múltiples formas a través de las cuales el alumnado pueda expresar su aprendizaje y; 3) Ofrecer diferentes formas de implicación por parte del alumnado, dando respuesta a las diferentes motivaciones que puedan existir entre las personas que conforman el grupo-clase. Cada uno de estos principios se relaciona con una red neuronal. Según las aportaciones de Segura y Quirós (2019) el primer principio se relacionaría con las redes de conocimiento, cuya función principal es disponer de la información, tratarla y clasificarla, asignándole un significado. El segundo de los principios estaría relacionado con las redes estratégicas, estas se relacionan con las acciones de planificar, programar, coordinar, poner en marcha acciones tanto motrices como mentales. En último lugar, el tercer principio, que hace referencia a las redes afectivas, relacionadas con la motivación de la persona por aprender.

El DUA supone una nueva manera de pensar la educación, un nuevo paradigma educativo, lejos de un enfoque tradicional donde la fuente del conocimiento y el saber queda de forma exclusiva en la figura del profesorado por otra, donde el alumnado es el protagonista de su aprendizaje y se parte de unas expectativas altas con respecto al alumnado y sus posibilidades. Permite responder a las diferentes experi- 


\section{$\begin{array}{lllllllllllll}\text { E } & \mathbf{X} & \mathbf{P} & \mathbf{E} & \mathbf{R} & \mathrm{I} & \mathrm{E} & \mathbf{N} & \mathrm{C} & \mathrm{I} & \mathrm{A} & \mathrm{S}\end{array}$}

encias previas, conceptos, ideas, valores, actitudes del alumnado, así como, favorecer la interacción. Igualmente conlleva una organización de aula y forma de trabajo diversa, en la que se trabajará tanto individual, como de forma grupal en pequeño y gran grupo, dependiendo de las necesidades del alumnado, del tema que se quiera abordar en clase y la forma en la que se desarrolle la misma. Con respecto a la evaluación, y teniendo en cuenta los planteamientos del DUA, Segura y Quirós $(2019, \mathrm{p} .7)$ no expone que supone un cambio, un repensar de las estrategias o herramientas a la hora de recoger la información del alumnado, puesto que:

- Deja de ser un mecanismo de poder que utiliza el personal docente para medir aprendizajes y se convierte en un trabajo de autorregulación a través de la reflexión personal y entre todas las personas implicadas en la mediación pedagógica.

- No se centra en pensar simplemente en los resultados de la evaluación sumativa, sino en ir reconfigurando la mediación pedagógica: los medios, las técnicas y los instrumentos que tradicionalmente se han utilizado, por otros donde el alumnado logre ser protagonista activo y aprendiz experto.

- El personal docente aplica una serie de medios y técnicas que les permite mediar el proceso de aprendizaje, con el fin de valorar los logros que paulatinamente obtienen, tomando decisiones e ir valorando el nivel competencial del estudiantado.

- Posibilita al alumnado demostrar sus aprendizajes a la vez que realiza una reflexión personal de sus propios procesos y crea nuevas rutas de estudio.

Son diversas las investigaciones que en los últimos años se han centrado en evaluar las metodologías bajo los principios del DUA (Ralabate, Currie-Rubin, Boucher y Bartecchi, 2014; McPherson, 2014; Sánchez, Díez y Martín, 2016) así como, la formación del profesorado, las cuáles han constatado la necesidad de una mayor sensibilización y formación (Marín, Berrocal y Campos, 2015) ante el desconocimiento de un diseño que ofrece la posibilidad de desarrollar prácticas que nos conduzcan hacia una inclusión efectiva. Por ello, la necesidad de formar y sensibilizar al alumnado universitario ante un diseño que le permitiría ajustar el proceso de enseñanza y aprendizaje a las características individuales de su alumnado.

\subsection{Neurodidáctica}

Todo lo expuesto anteriormente se relaciona con la Neurodidáctica, considerando a esta nueva disciplina como un fundamento sobre el que andamiar nuestras prácticas educativas y responder a lo planteado hasta el momento.

La neurodidáctica conocida también como neuroeducación, constituye un punto de convergencia entre el campo de la psicología, la sociología, la medicina y/o la neurociencia y la pedagogía, y se centra en aplicar los conocimientos del funcionamiento de nuestro cerebro al aprendizaje, buscando el desarrollo de todo su potencial (Forés y Ligioiz, 2009; Mora, 2017). Según Landívar (2012) "muestra un ángulo de la anatomía humana que ayuda a crear espacios educativos más asertivos puesto que se basa en el ser humano en sí mismo" (p.13). A continuación, sintetizamos en tres aspectos claves los planteamientos teóricos que la citada disciplina nos ofrece: 
1. Aprender en lo activo, es decir, haciendo, pues "el cerebro aprende y retiene mejor las informaciones, cuando el organismo tiene una intervención activa, se estimulan más sentidos y se comprenden más áreas en el proceso" (Ibarrola, 2013, p.108). Este postulado, fue puesto de manifiesto en los movimientos de renovación pedagógica de manos de autores y autoras como: Petalozzi, Dewey, Freinet, Montessori, etc. Mantenerse en lo activo, tiene mucho que ver con lo vivencial, donde es la propia persona inmersa en el proceso la que puede darle significado a eso que está aprendiendo (Cuesta, 2009), participando así de la construcción del conocimiento al que ha dotado de sentido, es decir, han entendido su/s finalidad/es, al enfrentarse a la resolución de situaciones problemáticas (López, 2018). De ahí, la necesidad e importancia de plantear retos reales y alcanzables en el aula (Tomlinson, 2005), teniendo en cuenta la transformación que experimenta el cerebro, en cuanto a su estructura y organización, desde los parámetros de la plasticidad cerebral (Cantó, 2015). Todo ello, permite aproximarse al conocimiento del entorno de una forma más real, dotando al alumnado de herramientas y estrategias básicas para la vida.

Este proceso de actividad en el individuo, siendo protagonista principal del proceso educativo, también está muy relacionado con que el aprendizaje nos tiene que permitir la transformación y no la mera reproducción de conocimiento e información, pues esto último no favorecería la evolución de la sociedad y de la humanidad. La transformación, lleva intrínseca un proceso de reflexión, pensamiento crítico, análisis, investigación, descubrimiento que poco o nada tiene que ver con el simple acto de memorizar que, relacionado con el aprendizaje, no es sinónimo del mismo.

Por último, hemos de tener en cuenta que, para mantenerse activa, la persona tiene que tener interés, motivación y curiosidad por lo que va a aprender, encontrando con ello relaciones con el siguiente planteamiento.

2. Aprender en lo emocional, desde la premisa de que las emociones son el motor de activación de los procesos cognitivos (Bisquerra, 2000; Casassus, 2006; Cuesta, 2009; Di Gesù y Seminara, 2012; Forés y Ligioiz, 2009; Ibarrola, 2013 y Mora, 2017); y es que "la curiosidad, el interés, el gozo y la motivación son fundamentales para aprender algo" (Cuesta, 2009, p. 32). El hecho de que el ámbito emocional tenga un espacio en nuestras aulas tiene como consecuencia, no sólo el favorecer el proceso de aprendizaje y de mejorar la convivencia, sino de dotar de competencias necesarias para la vida (Bisquerra, 2016). Ello se ha puesto de manifiesto por multitud de investigaciones tanto a nivel internacional como nacional (Mira, Parra y Beltrán, 2017; Soler, Aparicio, Díaz, Escolano y Rodríguez, 2016). Esto hace de la educación un proceso más humano (Esteve, 2003) que potencia la toma de conciencia de lo que se siente y cómo esto nos predispone de un modo u otro, no solo a pensar y a actuar, sino a vivir (Sánchez y Sánchez, 2016b), y por tanto a aprender. Así, se reconoce que "el ser humano funciona como un todo, de tal manera que lo cognitivo, está influenciado por lo lingüístico, por la afectividad y por el movimiento" (López, 2018, p.39) haciendo visible la interrelación y reciprocidad que existe entre 
los distintos ámbitos que nos componen como seres humanos (Sánchez y Sánchez, 2016a); pues como afirman Forés et al. (2015) "nuestro cerebro aprende de una manera integral y holística" (p.13).

Además de las emociones como promotoras de aprendizaje, se ha de tener en cuenta el ambiente o clima emocional en el que se desarrolla el mismo, pues éste debe estar caracterizado por seguridad, confianza, bienestar y respeto (Carpena, 2015; López, 2018; Tomlinson, 2005) como condicionantes emocionales que favorecen la exploración y el descubrimiento, base indispensable para el aprendizaje.

3. Aprender en lo cooperativo. Las distintas investigaciones que se han desarrollado a lo largo de estos últimos años (Azorín, 2018; Castro y Zurita, 2018; Saavedra, 2018) han constatado cómo el aprendizaje cooperativo en las distintas etapas educativas aporta numerosas ventajas al alumnado y su aprendizaje. Por un lado, permite al alumnado actuar sobre su propio proceso de aprendizaje de forma activa, supone una implicación por parte de todas las personas y asume su responsabilidad con respecto al resto y, por otro, desarrollar habilidades sociales, favoreciendo la escucha, respetando las aportaciones, ideas, visiones del resto de componentes y para lo que es necesario negociar, acordar, tomar decisiones consensuadas con el propósito de conseguir entre todas y todos un objetivo común. Aspectos que recoge la competencia social como una de las competencias emocionales que enuncia Bisquerra (2016). En palabras de Domingo (2008, p.237) el trabajo será cooperativo en la medida que se den los siguientes elementos: 1) En clase el alumnado aprendan en vez de que el docente enseñe; 2) Se trabaja con pequeños grupos en los que cada estudiante desempeña una función o rol; 3) Los/as estudiantes del grupo dependen necesariamente unos de otros; 4) Existe responsabilidad individual y de grupo; 5) Los estudiantes trabajan juntos, en un mismo espacio cercano; 6) Se desarrollan habilidades sociales y; 7) Los/as estudiantes reflexionan sobre su propia efectividad como grupo.

Además, debemos tener en cuenta que el acto de aprender "es una actividad social y cultural" (López, 2018) donde la cooperación se torna imprescindible para el desarrollo de los individuos, pues aprendemos de otras personas y con otras personas (Toro, 2005), ya que somos seres sociales (Sánchez y Sánchez, 2016a). Lo que nos permite, además, conocernos a nosotras y nosotros mismos (intrapersonal) y a la otredad (interpersonal), como parte de nuestro crecimiento individual y social. Sin obviar, que la acción de cooperar es antónima al individualismo y que, en ella, se producen emociones y sentimientos (López, 2018), lo que se encuentra muy relacionado con el postulado anterior.

\section{DESARROLLO DE LA EXPERIENCIA: ¿CÓMO ATENDEMOS A LA DIVERSIDAD EN LA UNIVERSIDAD?}

Antes de describir la experiencia que hemos llevado a cabo, consideramos necesario ofrecer cierta información que posibilite a la persona que lea este artículo poder situarse y aproximarse al contexto en el que se desarrolla la misma. 


\subsection{Contextualizando la experiencia}

La experiencia que ofrecemos en este trabajo se desarrolla en la Facultad de Ciencias de la Educación de la Universidad de Cádiz y se enmarca dentro del Grado de Educación Infantil, concretamente, en el último curso dentro la asignatura "Fundamentos Pedagógicos de las Necesidades Educativas en la Infancia". Como punto de partida consideramos relevante presentar los objetivos que guían nuestro quehacer docente:

- Por un lado, atender a la diversidad del alumnado matriculado en la asignatura, ya que como queda recogido en la Ley Orgánica 4/2007 del 12 de abril en su disposición adicional vigésimo cuarta, apartado 5: "Todos los Planes de Estudios propuestos por las universidades deben tener en cuenta que la formación [...] debe realizarse desde el respeto y la promoción de los Derechos Humanos y los principios de Accesibilidad Universal y Diseño para Todos".

- Por otro, ofrecerles durante esta formación inicial la información y conocimiento, así como las estrategias y competencias necesarias para aplicar en su futura práctica docente una educación inclusiva aplicando un diseño que permita la participación y el aprendizaje de todas las personas que forman parte del grupo-clase, desde la vivencia propia de metodologías didácticas y siendo conscientes de las posibles barreras que puedan frenar la accesibilidad haciendo que el entorno sea excluyente. De esta forma toman relevancia los procesos de investigación y reflexión continua que acompañan a la función docente.

- Por último, y no por ello menos importante, partimos de la necesidad de promover el encuentro entre las personas, dada la importancia de conocernos para entendernos, comprendernos y respetarnos desde ese compartir que nos une como compañeros y compañeras con retos y propósitos comunes. En su futura labor docente deberán construir redes de colaboración y espacios de trabajo con personas afines o no con las que deberán compartir y desarrollar proyectos, investigaciones en pro a la construcción de una educación inclusiva de calidad para la infancia; por ello, es de suma importancia que partan con una base desde su formación inicial en el compromiso con personas que desconocen y se esfuercen por entenderlas, comprenderlas para poder construir valores como la empatía, creando una cultura colaborativa en la que el dar y recibir recursos del entorno se hace imprescindible para un conocimiento más amplio de la realidad.

Para atender a estos objetivos expuestos en la propuesta docente que realizamos, donde se dilucida una intención clara de educación inclusiva en nuestras aulas, la diversidad no es solo respetada y atendida sino también celebrada como oportunidad de enriquecimiento. Para alcanzar dicha inclusión el equipo docente, de forma conjunta y coordinada, estableció una serie de estrategias didácticas y pedagógicas que atendiera a los fundamentos teóricos presentados en este escrito, y que se concretarían mediante: Trabajo por proyectos, Proyectos de Aprendizaje y Servicio, Aulas diversificadas, Aprendizaje Cooperativo, In- 
vestigación-acción, Tertulias Dialógicas, Talleres y Caza del tesoro, ésta última como dinámica más activa en la exposición de los trabajos realizados.

Cada año académico la asignatura se torna distinta como distinto es su alumnado, pues intenta atender al análisis de la realidad que nos encontramos en nuestras aulas y esto se produce en el momento en el que damos la bienvenida a nuestro alumnado. Como nos ofrece Barrio (2008):

La inclusión es un proceso, un plan abierto, una búsqueda interminable de formas de responder a la diversidad. Por tanto, no es un estado que se alcanza tras el logro de determinados objetivos, sino que se trata de un camino que emprenden las escuelas con el fin último de conseguir progresivamente que todos sus miembros se sientan parte integrante del centro, aceptados y bienvenidos (p.19).

Esta es nuestra intención y nuestra meta hacer de nuestras clases un aula acogedora y cada día más inclusiva. En esta ocasión, nos basaremos en las aulas diversificadas de Tomlinson (2001) ajustándose a nuestro contexto.

La elección de esta experiencia que engloba dos sesiones de trabajo en el aula, como describiremos a continuación, se debe a que es una experiencia vivenciada que alberga competencias que el alumnado puede transferir a sus futuras aulas de Educación Infantil donde podrán a través de ellas atender a la diversidad de su alumnado. Mediante esta actividad estaríamos dando respuesta al segundo y tercer principio del DUA, puesto que el alumnado, como veremos en las líneas sucesivas contará con múltiples formas a través de las cuales podrá expresar su aprendizaje, así como, decidir diferentes grados de implicación, atendiendo a las diferentes motivaciones que puedan existir. También se tienen en cuenta los principios de la neurodidáctica, pues se trata de un proceso de construcción en el que el alumnado está activo, trabaja de forma cooperativa en parejas o en tríos, y donde la motivación e interés lo despertamos con el planteamiento y desarrollo de la actividad en sí como un reto, creando un clima de confianza y seguridad en el aula.

Una vez contextualizada la situación de partida, pasaremos a detallar la puesta en práctica con el alumnado.

\section{2. ¿En qué consiste la experiencia?}

La experiencia como anticipamos en líneas anteriores, se desarrolla en dos sesiones de clase, concretamente de gran grupo, es decir, cuando está todo el grupoclase.

En primer lugar, una semana antes a la realización de la experiencia se le pide al alumnado que realice la lectura de un texto que se le facilitó a través del campus virtual de la asignatura. Como el grupo-clase se divide en dos subgrupos para la clase práctica, indicamos que cada uno de estos subgrupos realiza una lectura de un texto diferente, incluyendo al mismo tiempo dos de los ingredientes del trabajo cooperativo, concretamente la interdependencia positiva relacionada con la tarea a realizar y la responsabilidad individual (Moriña, 2008). 

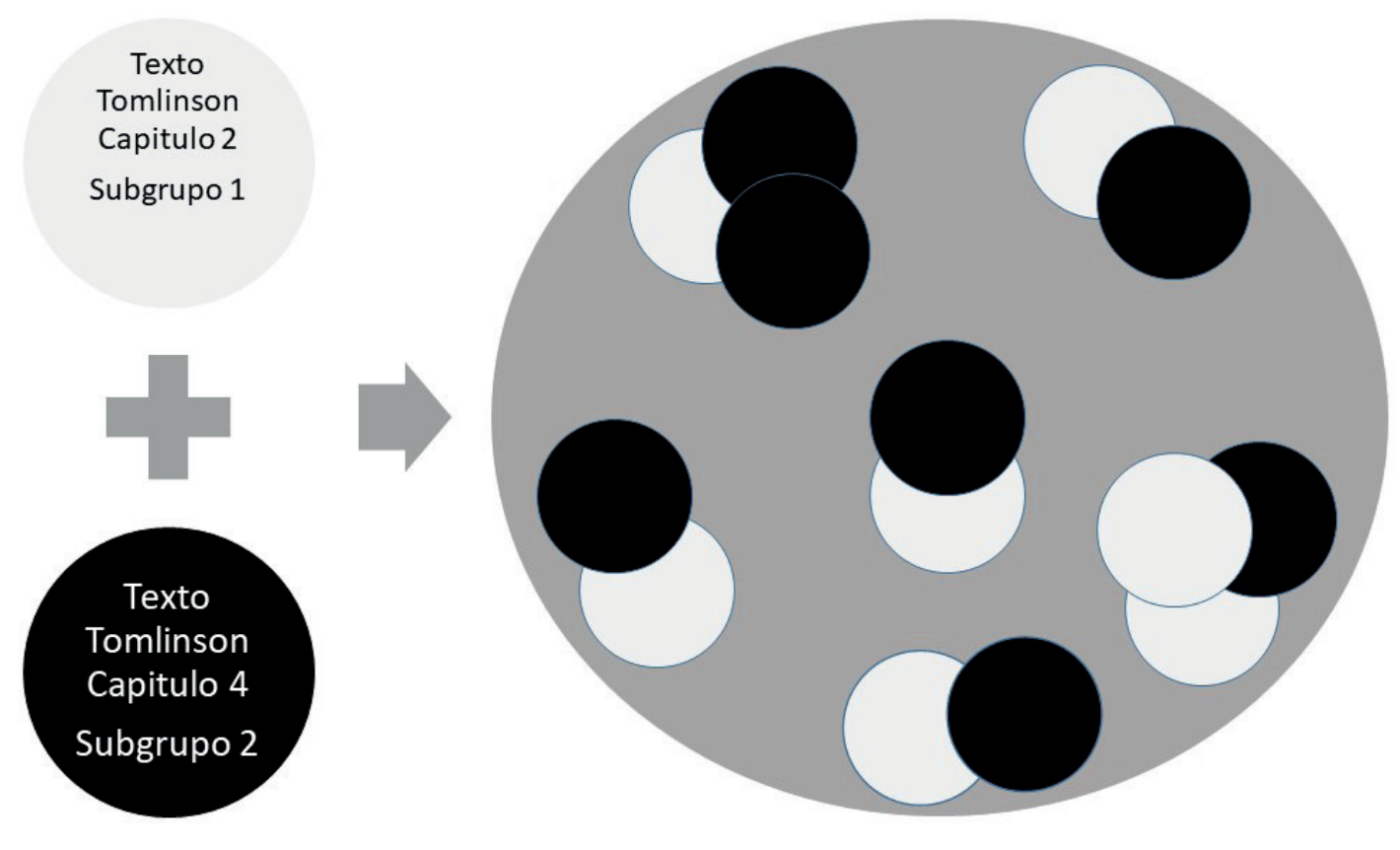

Figura 1: Comienzo y agrupamientos

En la sesión 1, una vez en clase se agrupan en parejas o tríos (dependerá del número total de asistentes a la sesión) como puede observarse en la Figura 1. Estos agrupamientos se realizaron de forma aleatoria a través de unas tarjetas numeradas previamente preparadas por las docentes para que cada persona tenga una pareja que haya leído un texto diferente. Esto se plantea en forma de juego, donde deben encontrar a la persona que tenga la misma tarjeta. Lo que va creando cierto clima lúdico que favorece la predisposición para la elaboración de la actividad en sí. Al mismo tiempo crea cierta incertidumbre que viene acompañada por emociones como la sorpresa, la intriga y la expectación de con quién me va a tocar trabajar hoy en clase. Con la premisa que existía la posibilidad de cambiar de pareja si la persona con la que le había tocado era su compañera o compañero habitual de trabajo y quisiera cambiar para trabajar con una persona con la que no había tenido la posibilidad de hacerlo con anterioridad.

Cuando cada persona ha encontrado a su pareja, se les plantea una actividad en forma de reto que deben resolver de forma cooperativa, ofreciéndoles la posibilidad de elegir el espacio más adecuado para realizar la actividad brindándoles la oportunidad de salir del aula (la terraza, cafetería, patio, biblioteca, salas de estudio). Introduciéndose de este modo, en un proceso de toma de decisiones con el que normalmente el alumnado no está familiarizado pues la mayor parte de las actividades se desarrollan dentro de clase. El ofrecerles espacios para que decidan, es para nosotras muy importante pues será la primera toma de decisiones que tendrán que hacer de forma conjunta, con una persona que quizás después de cuatro años de formación universitaria, es la primera vez que entabla comunicación. En segundo 
lugar, decidirán si salen o no de su zona de confort donde están cerca de su guía docente (aunque también nosotras nos movemos por los otros espacios), asimismo es una forma de conocer a nuestro alumnado, sus iniciativas, necesidades y sus intereses. También nos permite acercarnos a cómo realizan la gestión de los espacios y los recursos de los que disponen a su alcance, y qué efecto ejerce la presión académica y social sobre ellos y ellas y en qué medida.

El reto que se les plantea tras los agrupamientos es el siguiente: les pedimos que dialoguen e intercambien las ideas recogidas y trabajadas previamente de forma individual para así poder realizar un trabajo conjunto en el que evidenciar la construcción de un proyecto común. Para ello se les ofrece las siguientes posibilidades para que puedan elegir la que más se ajuste a sus intereses, sus necesidades y sus perfiles:

1. Elaboración de un decálogo en el que se muestren los principios y fundamentos recogidos en ambos textos leídos, al que deberán poner un título en función de lo que representa.

2. Diseño de una actividad concreta para un grupo-clase donde se vean que se respetan y atienden los principios y fundamentos recogidos en los textos leídos.

3. Búsqueda de un recurso que puede ser: vídeos, canciones, artículos de prensa, cuentos, imágenes, películas u otros, y que relacionen con los aspectos recogidos en el material trabajado, explicitando los nexos de unión que establecen.

4. Opción libre en la que pueden crear lo que quieran siempre que muestren evidencia de que han trabajado, comprendido y compartido la información expuesta en ambas lecturas, resultando de dicho trabajo una creación.

Se les ofrece todo el tiempo de la sesión que reste para la elaboración de la opción elegida. Durante el desarrollo y en relación al personal docente, este actuó en todo momento como guía del proceso, acercándose a las parejas o tríos, por si existían preguntas en relación a los textos, para facilitarles la comprensión de los mismos en caso de ser necesario, por si tenían dificultad en el seguimiento de la metodología o en el proceso de creación de la opción elegida.

En la sesión 2, se le facilita al alumnado los diez primeros minutos de la misma para que puedan acabar si les quedó pendiente algún aspecto a cerrar de la opción elegida. Principalmente esta sesión se centra en la exposición voluntaria de lo construido, en la que darán a conocer las ideas que han elaborado y desarrollado de los documentos trabajados. Para ello, se le ofreció la posibilidad de usar distintas herramientas, como: el retroproyector, póster, móviles, sólo oral, etc., todas las herramientas de las que disponemos en el aula para poder llevarla a cabo. Así mismo, se dividió la sesión de clase en segmentos de cuatro a seis minutos en los que las parejas fueron exponiendo sus trabajos y para la cual tenían que gestionar el orden de exposición, los tiempos, los silencios, las entradas, las actividades realizadas por la pareja o trío, etc. Entre los beneficios de dicha actividad, encontramos que permite que aquel grupo que lo desee pueda compartir sus ideas y todos los equipos aprenden de las aportaciones que ofrecen dichas exposiciones, enriqueciéndose de 
las visiones, conocimientos y herramientas usadas por las distintas parejas y tríos, teniendo como base el desarrollo de las habilidades comunicativas y la construcción desde un aprendizaje dialógico.

Una vez expuestas las aportaciones de las parejas o tríos, pasamos a realizar al alumnado una serie de cuestiones que se van respondiendo de viva voz en el grupo, continuando con la dinámica planteada en esta sesión. Lo que se persigue con las mismas es facilitar la reflexión y construcción desde la experiencia vivida. Las cuestiones fueron las siguientes:

- ¿Cómo os habéis sentido?

- ¿Qué os ha llevado a elegir la opción que habéis desarrollado?

- ¿Cómo ha sido el proceso de toma de decisiones?

- ¿Qué habéis aprendido de la dinámica en la que se ha desarrollado la actividad y de los textos leídos y trabajados?

- ¿Qué ventajas e inconvenientes le veis a la actividad?

- ¿Qué mejoras proponéis?

- ¿Qué metodología se pone en juego en esta actividad?

- ¿Qué relación establecéis entre la actividad propuesta y las ideas que recogen los textos leídos?

En relación a las preguntas planteadas al alumnado nos permite seguir construyendo desde enfoques diversos que nos aproximan a un mayor conocimiento del grupo tanto a nivel emocional como cognitivo, algo sumamente enriquecedor tanto para el grupo-clase como para el equipo docente.

\section{3. ¿Qué nos ofreció la experiencia?}

Tras la realización de las sesiones, la gran mayoría del alumnado eligió como primera opción de las descritas anteriormente, la elaboración de un decálogo. Las herramientas utilizadas para su exposición fueron diversas. En algunos casos, los equipos solamente lo leyeron, otros lo expusieron en Power Point y otros compartieron la información con sus compañeros y compañeras en forma de poesía o canción, todo ello atendiendo a su voluntad y a lo que previamente habían negociado y construido como equipo en la planificación de dicha exposición. Por tanto, en dicha recreación, prevalece el sentido del diseño universal para el aprendizaje, concretamente su tercer principio en el que se le asegura al alumnado múltiples formas de motivación, en las cuales se optimiza la elección y la autonomía y variando los niveles de desafío y apoyo (CAST, 2011).

La segunda opción más elegida fue la búsqueda de un recurso (vídeos, canciones, artículos de prensa, cuentos, imágenes, ...) todos ellos relacionados con los aspectos tratados en los documentos trabajados. De los recursos citados anteriormente, los que alcanzaron un mayor número de búsqueda entre el alumnado fueron los vídeos y las canciones, así como las imágenes. 
En tercer lugar, el alumnado optó por el diseño de una actividad concreta destinada a un grupo-clase y donde se incluyeran y se visibilizaran los principios y fundamentos recogidos en los textos leídos. Un ejemplo de ello fue el uso de un recurso multimedia para la recreación de la exposición de 10 ideas para promover la educación inclusiva, de modo que para ello utilizaron un video de un repositorio educativo online que reprodujeron a su grupo-clase. Posteriormente dividieron aleatoriamente al grupo en dos, otorgando dos roles distintos; una parte de la clase debería defender con argumentos contundentes coherentes y fundamentados por qué es importante apostar por la atención a la diversidad, la educación inclusiva y los apoyos dentro del aula y la otra parte de la clase debería hacer lo mismo, pero en contra. Por último, a modo de cierre hicieron un alegato de que aún se sentían poco preparados y preparadas para defender algo en lo que realmente creían y por lo que querían y pretendían apostar y volvieron a presentar un debate en relación a por qué creían que ocurría eso. Otras parejas o tríos, diseñaron actividades enfocadas a la Educación Infantil como la creación de ambientes o grupos interactivos.

La opción libre, aunque fue presentada a las parejas o tríos con bastante vehemencia, perseverancia, optimismo y altas expectativas, no fue elegida por ninguna.

En la Figura 2, se puede apreciar una representación de lo expuesto en líneas anteriores.

\section{Elegimos como pareja}
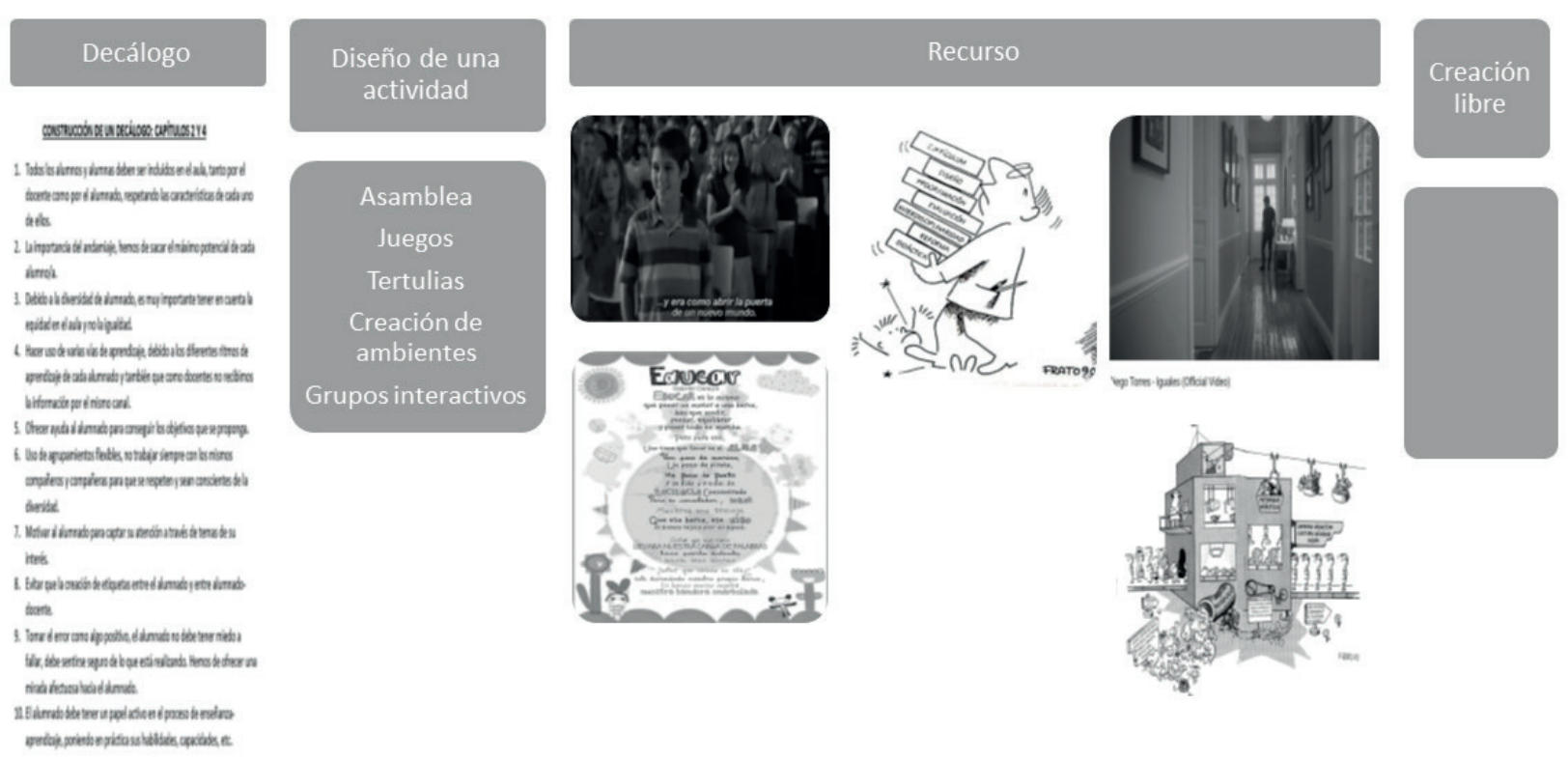

Figura 2: Muestra de algunos ejemplos de las creaciones

En cuanto a sus vivencias, percepciones, etc. que, en parte, se desprenden de las respuestas ofrecidas a las cuestiones que se realizaron y que se concretaron anteriormente podemos añadir que, para la mayoría fue una actividad en la que en general 
se sintieron cómodos y cómodas, destacando que al principio sí que sintieron cierto miedo por no saber con quién les iba a tocar y cómo iban a trabajar con esa persona. Y sorprendentemente, les había resultado fructífero y agradable trabajar con personas diferentes a las habituales además de una oportunidad para interactuar con compañeros y compañeras con las que, a pesar de convivir como grupo durante cuatro años, no habían cruzado una palabra o ni siquiera sabían cómo se llamaban. Algo que también les sorprendió fue el hecho de que les preguntáramos, en primer lugar, cómo se habían sentido, al ser algo que no se suele realizar en las aulas o que hayan experimentado cotidianamente en su trayectoria escolar. Otro aspecto que también llamó su atención y pusieron de manifiesto fue el elevado número de parejas que habían decidido elaborar el decálogo a lo que añadieron que a pesar de estar continuamente quejándose y reclamando que se les ofrezca más de una opción a la hora de realizar una actividad, cuando se le da la oportunidad, se quedan con la opción más próxima a su zona de confort, es decir, a lo que dominan y están acostumbrados y acostumbradas a realizar y no se aventuran a probar opciones o posibilidades nuevas. Fue muy significativo, como al preguntarles sobre la relación entre la actividad propuesta y las ideas que recogen los textos leídos, toda la clase dijo que a través de la actividad planteada se podían identificar todos los principios y fundamentos recogidos en los citados textos. En lo referente al aprendizaje manifestaron el desarrollo de ciertas competencias emocionales, concretamente la más vinculadas con la competencia social (Bisquerra, 2016), con aspectos como: habilidades comunicativas (de expresión y de recepción), negociación, respeto, trabajo en equipo, etc. Para finalizar con este aspecto, hicieron hincapié en el valor de las actividades desarrolladas en la experiencia para su futuro, pues manifestaban sentir poder realizarla con el alumnado de infantil tras haberlo realizado ellos y ellas mismas y haber tenido la oportunidad de reflexionar sobre ella. Incluso aludieron a la famosa frase de Confucio "Lo escuché y lo olvidé, lo vi y lo entendí, lo hice y lo aprendí", algo que como docentes nos llena de satisfacción.

\section{ANÁLISIS Y REFLEXIONES}

Se puede apreciar en la experiencia como queda reflejada una respuesta de las docentes a todas las necesidades del alumnado atendiendo a todos los canales posibles que presentan, así mismo, como se llevan a cabo distintas tareas adecuadas a la edad, al contexto, a las capacidades y a los intereses del alumnado, creando grupos flexibles y evaluando de forma continua y posibilitando el ajuste continuo. En el caso de dicha experiencia se ha diversificado el proceso y el producto de acuerdo con las aptitudes, los intereses y el perfil del aprendizaje gracias a las estrategias organizativas basándonos en los principios del diseño universal para el aprendizaje. Aunque la actividad comenzó en forma de texto, esta podía ser modificada ajustándose a las parejas y tríos a través de diferentes canales ya fueran visuales, auditivos o kinestésicos, al ofrecerles distintas vías de representación de la actividad.

Una vez más el ofrecer distintas posibilidades facilitó la accesibilidad y permitió respetar y responder a la diversidad como un valor añadido, y es que atender a la di- 
versidad no es más que ocuparse y satisfacer "a todas las exigencias de la naturaleza humana para conseguir que cada hombre o mujer llegue a ser la mejor persona posible, en todas sus notas constitutivas y dimensiones" (Bernardo, Javaloyes y Calderero, 2007, p.17). Para ello, debemos personalizar la enseñanza, conocer a nuestro alumnado y que nuestro alumnado se conozca. Estrategias como la de las aulas diversificadas nos favorecen esta labor pues, por un lado, nos acerca al alumnado, a sus intereses y a sus necesidades y, por otro, también lo hace a sus compañeros y compañeras facilitándoles el apoyo y la ayuda mutua entre iguales, promoviendo comportamientos prosociales y cooperativos, otro aspecto dentro de la competencia social perteneciente a las competencias emocionales (Bisquerra, 2016) y que evidencia principios de la neurodidáctica.

Por último, nos parece significativo como punto de reflexión resaltar que la última opción ofrecida al alumnado no tuviera ninguna aceptación por parte del mismo. Desconocemos si es falta de creatividad, miedo a salir de su zona de confort o el mal hábito aprendido año tras años, realizando actividades donde dicha creatividad ha sido mermada por directrices demasiado marcadas (algo manifestado por el alumnado en actividades anteriores) y que podrían hacer que se sintieran incómodas o incómodos en espacios poco controlados en los que no estaban acostumbradas/os a moverse. Son libres para crear, son libres para pensar, son libres para reflexionar y construir algo nuevo y distinto, sin embargo, eligen no hacerlo. Sería interesante analizar con profundidad si es realmente una decisión tomada o el producto de un proceso enseñado, pues también muchas parejas o tríos optaron por no salir del aula, aun teniendo la posibilidad de hacerlo.

\section{A MODO DE CIERRE}

Diseñar programas formativos donde se tenga en cuenta las necesidades, inquietudes e intereses permite promover oportunidades de aprendizaje para todos y todas, así como, mejorar el desarrollo profesional del alumnado universitario, propiciando a su vez esos cambios tan necesarios en las políticas, culturas y prácticas de nuestro sistema educativo que respalde la equidad en educación evitando y derribando los entornos excluyentes con los que convivimos a diario y que frenan y merman el desarrollo integral, global y holístico de todas las personas que formamos parte de la sociedad y que nos caracterizamos por ser diversas. La experiencia realizada, y que ha sido presentada a lo largo de este trabajo, ha permitido al alumnado reflexionar y analizar desde su propia vivencia y experiencia en qué medida nuestras actitudes (positivas o negativas) como figura docentes, la percepción y expectativas que ofrecemos con respecto a nuestro alumnado, así como ofrecer o no diversas alternativas para acceder a la información y representar la construcción de sus conocimientos, puede favorecer o aminorar las desigualdades de partida posibilitando así, la eliminación de barreras al aprendizaje y la participación a favor de alcanzar la justicia social. Vivirla en primera persona hace posible la experimentación por parte del alumnado de una metodología inclusiva que en sesiones anteriores habían manifestado como puntos de interés puesto que se sentían reacios y reacias a las posibilidades 
de su ejecución. Esto les hace acercarse a esta metodología inclusiva siendo agentes activos del proceso de transformación que en está en sus manos como futuras y futuros docentes para hacer posible "el derecho a la diferencia pero nunca la diferencias de derechos" (Torres, 2008, p.109).

Con este trabajo, pretendemos hacer visible que la diversidad es una realidad que está presente en nuestras aulas, tanto a nivel universitario como en educación infantil, primaria, secundaria, y que al tratarse de un aspecto intrínseco a la condición humana, consideramos imprescindible, indispensable, esencial y necesario que en la formación inicial del profesorado se trabaje desmontando esa visión utópica que pone de relieve al alumnado cuando se habla de una pedagogía inclusiva que respete, atienda y valore lo que realmente somos: diversidad. Y no entendemos mejor forma de llevarlo a cabo que hacérselo sentir, vivir y construir juntos y juntas.

\section{REFERENCIAS}

Aguilar, S. y Benítez, R. (2017). Investigando desde una perspectiva inclusiva el uso de las TIC como recurso de acceso al aprendizaje y atención a la diversidad del alumnado mayor. El profesorado universitario y su formación en diseño universal de aprendizaje (CD-ROM). En Ruiz-Palmero, J., Sánchez-Rodríguez, J. y Sánchez-Rivas, E. (Edit.), Innovación docente y uso de las TIC en educación (p.106). Málaga: UMA Editorial.

Alba, C. (Coord.) (2016). Diseño universal para el aprendizaje: educación para todos y prácticas de enseñanza inclusivas. Madrid: Morata.

Alba, C. (2018). Diseño Universal para el Aprendizaje un modelo didáctico para proporcionar oportunidades de aprender a todos los estudiantes. Padres y Maestros, (374), 21-27. DOI: pym.i374.y2018.003

Alba, C., Sánchez, J. M. y Zubillaga, A. (2014). Diseño Universal para el Aprendizaje (DUA). Pautas para su introducción en el currículo. Madrid: Edelvives.

Alba, C., Sánchez, P., Sánchez, J. M. y Zubillaga, A (2015). Pautas sobre el Diseño Universal para el Aprendizaje (DUA).Texto completo (Versión 2.0). Recuperado de:http://www. udlcenter.org/sites/udlcenter.org/files/UDL Guidelines v2.0full

Azorín, C. Mạ. (2018). El método de aprendizaje cooperativo y su aplicación en las aulas. Perfiles Educativos, XL (161), 181-194.

Bächler, R. y Pozo, J.I. (2016). ¿Siento, luego enseño? Concepciones docentes sobre las relaciones entre las emociones y los procesos de enseñanza/aprendizaje. Infancia y Aprendizaje, 39 (2), 312-348. doi: https://doi.org/10.1080/02103702.2015.1133088.

Barrio, J.L. (2009). Hacia una Educación Inclusiva para todos. Revista Complutense de Educación, 20 (1),13-31.

Benítez, R. y Aguilar, S. (2015). Entornos virtuales para atender a la diversidad. Hachetetepé. Revista de Educación y Comunicación, (10), 97-106.

Bernardo, J., Javaloyes, J.J. y Calderero, J.F. (2007). Cómo personalizar la educación. Madrid: Narcea.

Bisquerra, R. (2000). Educación emocional y Bienestar. Barcelona: CISS Praxis.

Bisquerra, R. (2016). 10 ideas claves. Educación Emocional. Barcelona: Graó. 
Bravo, I. y Herrera, L. (2011). Convivencia escolar en Educación Primaria. Las habilidades sociales del alumnado como variable moduladora. DEDiCA. Revista de EducaÇÃo e humanidades, 1, 173-212.

Carpena, A. (2015). La empatía es posible. Educación emocional para una sociedad empática. Bilbao: Desclée de Brouwer.

Casassus, J. (2006). La educación del ser emocional. México: Castillo.

CAST (Center for Applied Special Technology) (2011). Universal Design for Learning Guidelines. Version 2.0. Wakefield, MA: Author.

Castro, M. y Zurita, F. (2018). Aprendizaje cooperativo. Propuesta de intervención en Educación Primaria. Trances. Transmisión del Conocimiento Educativo y de la Salud, (2), 137-152.

Contó, J. (2015). Resultados de la implementación de la neurodidáctica en las aulas de educación infantil. Opción: Revista de Ciencias humanas y sociales, (5), 189-199.

Cuesta, J. (2009). Neurodidáctica y estimulación del potencial innovador para la competitividad en el tercer milenio. Revista Educación y desarrollo social, 3 (2), 28-35.

Diez, A. y Huete, S. (1997). Educar en la diversidad. Educar hoy, (60), 15-17.

Di Gesù, F. y Seminara, A. (2012). Neurodidáctica y la implicación de las emociones en el aprendizaje. Lynx: Panorámica de Estudios Lingüísticos, (11), 5-39.

Domingo, J. (2008). El aprendizaje cooperativo. Cuadernos de Trabajo Social, 21, 231-246.

Esteve, J.M. (2003). La tercera revolución educativa. Barcelona: Paidós.

Forés, A y Ligioiz, M. (2009). Descubrir la neurodidáctica. Barcelona: UOC.

Forés, A., Gamo, R., Guillén, J.C., Herández, T., Ligioiz, M., Pardo, F. y Trinidad, C. (2015). Neuromitos en educación. El aprendizaje desde la neurociencia. Barcelona: Plataforma actual.

Giné, C. y Font, J. (2007). El alumnado con discapacidad intelectual y del desarrollo. En Bonals, J. y Sánchez, M. (Coord.), Manual de asesoramiento psicopedagógico (pp. 879914). Barcelona: Grao.

Gónzalez, M.M., Díez, M., López, F. y Román, M. (2010). La importancia del clima emocional del aula desde la perspectiva del alumnado universitario. Revista de Enseñanza Universitaria, 35, 16-27.

Ibarrola, B. (2013). Aprendizaje emocionante: neurociencia para el aula. Madrid: Fundación SM.

Landívar, A. M. (2012). Neuroeducación: Educación para jóvenes bajo la lupa de María Montessori. Córdoba: Brujas.

López, M. (2018). Fundamentos y prácticas inclusivas en el Proyecto Roma. Madrid: Morata.

Ley Orgánica 4/2007, de 12 de abril, por la que se modifica la Ley Orgánica 6/2001, de 21 de diciembre, de Universidades. Boletín Oficial del estado, de 13 de abril de 2007, núm. 89, páginas 16241 a 16260.

Marín, M.G., Berrocal, V. y Campos, J. (2016). La aplicación de los principios del Diseño Universal del Aprendizaje en un elemento curricular de un modelo de educación a distancia. En Roig, R. (coord.), Tecnología, innovación e investigación en los procesos de enseñanza-aprendizaje (pp. 2647-2658). Barcelona: Octaedro. 
McPherson, S. (2014). Strategies and resources for preparing teachers for STEM teaching and learning. En Searson, M. y Ochoa, M. (Eds.), Proceedings of society for information technology \& teacher education international conference 2014 (pp. 1927-1939). Chesapeake, VA: AACE.

Mira, J. G.; Parra, M. C. y Beltrán, M. A. (2017). Educación emocional en la universidad: propuesta de actividades para el desarrollo de habilidades sociales y personales. Vivat Academia, Revista de comunicación, 139, 1-17. DOI: http://doi.org/10.15178/ va.2017.1-17.

Mora, F. (2017). Neuroeducación. Solo se puede aprender aquello que se ama. Madrid: Alianza.

Moriña, A. (2008). La escuela de la diversidad. Materiales de formación para el profesorado. Madrid: Editorial Síntesis.

Pérez, A.I. (2014). Aprender a pensar para poder elegir. Cuadernos de Pedagogía, (447), 38-41.

Ralabate, P., Currie-Rubin, R., Boucher, A. y Bartecchi, J. (2014). Collaborative planning using universal design for learning. Perspectives on School-Based Issues, 15, 26-31. doi:10.1044/sbi15.1.26

Saavedra, M.C. (2018). Aprendizaje Cooperativo basado en la Investigación en la Educación Superior. REDU. Revista de Docencia Universitaria, 16(1), 235-250

Sánchez, S., Díez, E. y Martín, R. A. (2016). El diseño universal en la educación como medio para atender a la diversidad. Una revisión de casos de éxito en la universidad. Contextos Educativos: Revista de Educación, 19, 121-131. doi:10.18172/con.2752

Sánchez, A. y Sánchez, L. (2016a). Antiprograma de Educación Emocional. Sentir en primera persona. Madrid: Wolters Kluwer.

Sánchez, L. y Sánchez, A. (2016b). La educación emocional: un cambio de mirada. Una propuesta desde lo emergente y vivencial. En M. Fernández y N. Alcaraz (Coords.), Innovación educativa. Más allá de la ficción (pp. 159-172). Madrid: Pirámide.

Segura, M.A. y Quirós, M. (2019). Desde el Diseño Universal para el Aprendizaje: el estudiantado al aprender se evalúa y al evaluarle aprende. Revista Educación, 43 (1), 1-13. DOI: https://doi.org/doi:10.15517/revedu.v43i1.28449

Soler, J.L., Aparicio, L., Díaz, O., Escolano, E. y Rodríguez, A. (Coords.) (2016). Inteligencia emocional y bienestar II. Reflexiones, experiencias profesionales e investigaciones. Zaragoza: Ediciones Universidad San Jorge.

Tomlinson, C.A. (2001). Elementos constitutivos de la diversificación y Estrategias docentes que apoyan la diversificación. En Tomlinson, C.A., El Aula Diversificada. Dar una respuesta a las necesidades de todos los estudiantes (pp.29-40). Barcelona: Octaedro.

Tomlinson, C.A. (2005). Estrategias para trabajar con la diversidad en el aula. Buenos Aires: Paidós.

Tomlinson, C.A. (2013). El aula diversificada: dar respuesta a las necesidades de todos los estudiantes. Barcelona: Octaedro.

Tonucci, F. (2017). A modo de introducción... La diversidad como valor en una escuela que cambia. Aula Abierta, (46), 9-12. DOI: https://doi.org/10.17811/rifie.46.2017.9-12

Toro, J.M. (2005). Educar con “co-razón”. Bilbao: Desclée de Brouwer.

Torres, J. (2008). Diversidad cultural y contenidos escolares. Revista de Educación, (345), 88-110. 